\title{
Robust Optimal Control Applied to a Composite Laminated Beam
}

\author{
Edson Hideki Koroishi , Fabian Andres Lara Molina , Albert Willian Faria², Valder Steffen Junior
}

\begin{abstract}
This paper proposes an active vibration control technique, which is based on linear matrix inequalities, that is applied to a piezoelectric actuator bonded to a composite structure, forming a so-called smart composite structure. Serendipity-type finite element, based on first-order shear deformation theory with rectangular shape, eight nodes, five mechanical degrees of freedom (DOF) per node and eight electrical DOF per piezoelectric layer, is established for the composite structural model. Additionally, a mixed theory that uses a single equivalent layer for the discretization of the mechanical displacement field and a layerwise representation of the electrical field is adopted. Temperature effects are neglected. Simulation results illustrate the effectiveness of the proposed vibration control methodology for composite structures.
\end{abstract}

KEYWORDS: Composite materials, PZT actuator, Robust control, Linear matrix inequalities.

\section{INTRODUCTION}

The recent years have seen the appearance of innovative materials, as the so-called composite materials, particularly in aerospace applications. The structures constructed by this innovated arrangement are characterized by lightness, high mechanical resistance, and the possibility to be optimized for a specific working condition. Unlike the regular materials (steel, aluminum etc.), the composites are formed by various layers with different fiber orientations, which allows to be tailored for a particular application (Reddy, 1997). Aircraft, aerospace and automotive industries are examples in which the composite materials have been increasingly used.

Generally, piezoelectric layers (PZT sensor/actuator patch) are incorporated to the composite materials in order to offer potential benefits in a wide range of applications, such as structural health monitoring, noise suppression, precision positioning and active vibration control (Thinh and Ngoc, 2010). Thus, the set encompassing the composite material, piezoelectric layers and monitoring and control systems is known as Smart Composite Structure.

Techniques have been developed to represent the dynamic behavior of smart composite structures, as the so-called Mixed Theory, that uses a single equivalent layer for the discretization of the mechanical displacement and a layerwise concept for the electrical field (Saravanos et al., 1997). Both mechanical and electrical fields are incorporated to the finite element formulation through the Hamilton's variational principle, leading to sets of equations that are solved by using appropriated boundary conditions.

Basically, the mechanical field is represented by applying two main theories: First-order Shear Deformation Theory (FSDT) and Higher-order Shear Deformation Theory (HSDT).

1.Universidade Tecnológica Federal do Paraná - Cornélio Procópio/PR - Brazil 2.Universidade Federal do Triângulo Mineiro - Uberaba/MG - Brazil 3.Universidade Federal de Uberlândia - Uberlândia/MG - Brazil.

Author for correspondence: Edson Hideki Koroishi| Department of Mechanical Engineering - Universidade Tecnológica Federal do Paraná | Avenida Alberto Carazzai, 1.640 - Centro | CEP: 86.300-000 - Cornélio Procópio/PR - Brazil | Email: edsonh@utfpr.edu.br

Received: 07/14/2014 | Accepted: 02/01/2015 
These theories have favorable and unfavorable characteristics, especially regarding the accuracy, scope and computational effort involved in their implementation (Reddy, 1997; Chee, 2000).

FSDT takes into account a constant distribution of transverse shear strain through the laminate thickness. In accordance with real situation, the distribution is parabolic, being necessary to introduce a constant correction in FSDT (Reddy, 1997; Cen et al., 2002). HSDT is well adapted to represent both thin and thick laminated composite plates. Also, it takes into account a parabolic distribution of transverse shear strains without requiring any correction, such as in the FSDT approach. However, HSDT presents an increased analytical framework and a higher number of degrees of freedom as compared with the FSDT, which represents higher computational cost. Also, FSDT generates precise mechanical characteristics, as deflections, natural frequencies and buckling loads (Lima et al., 2010).

In the context of countless demands of mechanical systems with optimal performance, this work proposes to design an active vibration control architecture for a simple smart composite structure (clamped-free smart beam). In this study, the mixed theory is used to represent the dynamic behavior of the structure. The linear quadratic regulator (LQR) approach, solved by linear matrix inequalities (LMI), allows for the calculation of the feedback controller's gain.

LMI is a useful tool for constrained problems in which the parameters vary according to a range of values. Once formulated in terms of LMI, the problem can be solved efficiently by convex optimization algorithms (Boyd et al., 1994). The advantage of using LMI for determining the controller gain is the possibility of assuming that the parameters of the model involve uncertainties. Then, a robust vibration control system can be designed. In general, the layer thicknesses, the fiber orientations and the elasticity modulus of the composite structure are some of the parameter uncertainties to be taken into account.

Additionally, the balanced realization method is used to reduce the complexity of the computational model for only the most important vibration modes of the structure in order to increase the efficiency of the controller.

\section{COMPOSITE MATERIAL}

\section{MECHANICAL DISPLACEMENT FIELD OF THE MIXED THEORY}

In the Mixed Theory, the mechanical behavior of the structure is represented by a first order field (FSDT), expressed as:

$$
\begin{aligned}
& u(x, y, z, t)=u_{0}(x, y, t)+z \Psi_{x}(x, y, t) \\
& v(x, y, z, t)=v_{0}(x, y, t)+z \Psi_{y}(x, y, t) \\
& w(x, y, z, t)=w_{0}(x, y, t)
\end{aligned}
$$

where:

$u_{0}, v_{0}$ and $w_{0}$ : displacements in the directions $x, y$ and $z$, respectively, addressed to a material point of the mean reference plane $(x-y)$;

$\Psi_{x}$ and $\Psi_{y}$ : rotations around the $x$ and $y$ axis, respectively, of the orthogonal segments to the reference surface.

The mechanical variables presented in Eq. 1 are described by finite elements by using appropriated shape functions and nodal variables (mechanical variables). The element considered in the formulation is known as serendipity, i.e. a plate element with three nodes per edge in a total of eight (Reddy, 1997).

The mechanical displacement field described by FSDT, rewritten in local elementary coordinates, is given by:

$\{U(\xi, \eta, z, t)\}=\left[A_{u}(z)\right]\left[N_{u}(\xi, \eta)\right]\left\{u_{e}(t)\right\}$

where:

$\{U(\xi, \eta, z, t)\}=\{u(\xi, \eta, z, t) v(\xi, \eta, z, t) w(\xi, \eta, z, t)\}^{T}$

$\left[A_{u}(z)\right]$ : matrix of the thickness variables $z$ from five in-plane functions $\left(u_{0}, v_{0}\right.$ and $w_{0}, \Psi_{x}$ and $\left.\Psi_{y}\right)$;

$\left\{u_{e}(t)\right\}$ : vector that contains all nodal variables;

$\left[N_{u}(\xi, \eta)\right]$ : matrix of mechanical shape functions.

The mechanical deformation is presented in terms of the shape functions and nodal displacement, as shown by Eq. 3 .

$\{\varepsilon(\xi, \eta, z, t)\}=\left[B_{u}(\xi, \eta, z)\right]\left\{u_{e}(t)\right\}$

where:

$\left[B_{u}(\xi, \eta, z)\right]=[D(z)]\left[N_{u}(\xi, \eta)\right]$;

$[D(z)]$ : matrix formed by differential operators appearing in the strain-displacement relations, as detailed in (Chee, 2000).

\section{LINEAR ELECTRIC POTENTIAL DISTRIBUTED IN THE LAYERS}

The approximation of the electric potential expressed in terms of the Mixed Theory is given by Eq. 4 . Note that the coordinate $z$ in the thickness direction of the plate is decoupled with respect to the reference surface coordinates $(x-y)$. 
$\varphi(x, y, z, t)=\sum_{j=1}^{n c+1} L_{j}(z) \varphi_{j}(x, y, t)$

where:

$L_{j}(z)$ : layerwise function;

$\varphi_{j}(x, y, t)$ : interface functions of the $j^{\text {th }}$ composite interface constituted by $n c$ layers.

The electric potential described in local coordinates for the $k^{\text {th }}$ elementary layer of the $e^{\text {th }}$ element is expressed in the finite element formulation as shown in Eq. 5:

$\varphi(\xi, \eta, z, t)^{\mathrm{k}}=\left[N_{\varphi}(\xi, \eta, z)\right]\left\{\varphi_{e}(t)\right\}$

where:

$\left[N_{\varphi}(\xi, \eta, z)\right]$ : matrix of electric shape functions incorporating the serendipity shape functions and the Lagrangian interpolating functions;

$\left\{\varphi_{e}(t)\right\}$ : contains the nodal values of electric potential.

Using the definition for the electric field as the negative gradient of the electric potential and by taking into account Eq. 5, the expansion of the electric field for the $k^{- \text {th }}$ layer is expressed by:

$\left\{E(\xi, \eta, z, t)_{\mathrm{e}}^{\mathrm{k}}\right\}=\vec{\nabla}\left(\left[N_{\varphi}(\xi, \eta, z)\right]\left\{\varphi_{e}(t)\right\}\right)$

where:

$\vec{\nabla}\left(\left[N_{\varphi}(\xi, \eta, z)\right]\right.$ are the effective electric shape function matrix (Chee, 2000);

$B_{\varphi}$ : input matrix.

\section{FORMULATION OF THE ELEMENTARY MATRIX}

The coupling between the composite structure and the piezoelectric element is made through the Hamilton's variational principle, that incorporates all energy contributions presented in the structure. According to Chee et al. (2000), the coupling elementary matrices are the following:

$$
\begin{aligned}
& {\left[M^{e}\right]=\int_{V e} \rho\left[N_{u}\right]^{T}\left[A_{u}\right]^{T}\left[A_{u}\right]\left[N_{u}\right] d V_{e}} \\
& {\left[K_{u u}^{e}\right]=\sum_{k=1}^{n c} \int_{\xi=-1}^{+1} \int_{\eta}^{+1} \int_{z=z_{k}}^{z_{k+1}}\left(\left[B_{u}\right]^{T}[c]\left[B_{u}\right]\right) J d z d \eta d \xi} \\
& {\left[K_{u \varphi}^{e}\right]=\sum_{k=1}^{n c} \int_{\xi=-1}^{+1} \int_{\eta}^{+1} \int_{z=z_{k}}^{z_{k+1}}\left(\left[B_{u}\right]^{T}[e]^{T}\left[B_{\varphi}\right]\right) J d z d \eta d \xi}
\end{aligned}
$$

$$
\left[K_{\varphi \varphi}^{e}\right]=\sum_{k=1}^{n c} \int_{\xi=-1}^{+1} \int_{\eta}^{+1} \int_{z=z_{k}}^{z_{k+1}}-\left(\left[B_{\varphi}\right]^{T}[\chi]\left[B_{\varphi}\right]\right) J d z d \eta d \xi
$$

where:

$\rho:$ material density;

$\left[M^{e}\right]$ : elementary mass matrix;

$\left[K_{u u}^{e}\right]$ : elementary matrix of elastic stiffness;

$\left[K_{u \varphi}^{e}\right]$ : stiffness elementary matrices of electromechanical coupling;

$\left[K_{\varphi \varphi}^{e}\right]$ : known as dielectric elementary matrix;

$[c],[e]$ and $[\chi]$ : respectively, the elastic stiffness, piezoelectric stress and electric permissivity matrices of constant values;

$\left[B_{u}\right]$ : input matrix;

$V_{e}$ : elementary volume;

$J$ : the Jacobian of the transformation (Reddy, 1997).

Equation 11 shows the global matrices of the model constructed through the standard procedure, in which the subscript $g$ indicates global quantities.

$\left[\begin{array}{cc}{\left[M_{g}\right]} & 0 \\ 0 & 0\end{array}\right]\left\{\begin{array}{l}\left\{\ddot{u}_{g}\right\} \\ \left.\ddot{\phi}_{g}\right\}\end{array}\right\}+\left[\begin{array}{cc}{\left[K_{u u}\right]} & {\left[K_{u \varphi}\right]} \\ {\left[K_{\varphi u}\right]} & {\left[k_{\varphi \varphi}\right]}\end{array}\right]\left\{\begin{array}{l}\left\{u_{g}\right\} \\ \left\{\phi_{g}\right\}\end{array}\right\}=\left\{\begin{array}{l}\left\{F_{g}\right\} \\ \left\{Q_{g}\right\}\end{array}\right\}$

where:

$M_{g}$ : global matrix elementary;

$\ddot{u}_{g}$ : acceleration vector;

$\ddot{\varnothing}_{g}$ : second derivative of $\phi_{g}$;

$u_{g}$ : displacement vector;

$\phi_{g}$ : vector of electrical potential;

$\left\{F_{e}\right\}$ and $\left\{Q_{e}\right\}$ : respectively, the generalized force and nodal charge (elementary).

\section{BALANCED REALIZATION}

Balanced realization consists in describing the model of the system in the space state form and combining the controllability and observability matrices for each state of the system by using the Gramians of controllability and observability of the system. The linear transformation that leads the system to this representation is called balanced transformation. In this method, the reduced model is obtained by neglecting the states associated with the small singular values (Assunção and Hemerly, 1992; Laub et al., 1987).

A balanced realization is an asymptotically stable minimal realization in which the controllability and observability Gramians 
are equal and diagonal (Conceição et al., 2009). Consider a stable linear-time invariant system defined by the state space equations:

$$
\begin{aligned}
& \{\dot{x}(t)\}=[A]\{x(t)\}+[B]\{u(t)\} \\
& \{y(t)\}=[C]\{x(t)\}
\end{aligned}
$$

where:

$\dot{x}(t)$ : derivative of $x(t)$ with respect to time;

$x(t)$ : state vector;

$[A]: n \times n$ dynamic matrix;

$[B]: n \times m$ input matrix;

$[C]: s \times n$ output matrix;

$\{u(t)\}:$ input force;

$\{y(t)\}$ : output vector;

$n$ : order of the system;

$m$ : number of inputs;

$s$ : number of outputs.

The system is called balanced if the solutions to the following Lyapunov equations (Eq. 14 and Eq. 15$)$ are $\mathrm{P}=\mathrm{Q}=\operatorname{diag}\left(\sigma_{1}, \sigma_{2,}, \ldots, \sigma_{\mathrm{n}}\right)$ :

$$
\begin{gathered}
{[A][P]+[P][A]^{T}+[B][B]^{T}=0} \\
{[A]^{T}[Q]+[Q][A]+[C]^{T}[C]=0}
\end{gathered}
$$

where:

$[P]$ and $[Q]$ : respectively, the controllability and observability Gramians;

$\sigma_{\mathrm{i}}(i=1,2, \ldots, n):$ the singular values of the system $\left(\sigma_{1} \geq \sigma_{2} \geq \ldots \geq \sigma_{n} \geq 0\right)$.

Every $\sigma_{\mathrm{i}}$ is associated with a state $x_{\mathrm{i}}$ of the balanced system. Its value quantifies the contribution that $x_{\mathrm{i}}$ makes to the input-output behavior of the system. As $\sigma_{1} \geq \sigma_{2}$, then $x_{1}$ affects the behavior of the system more than $x_{2}$, due to the fact that the singular values are ranked from the most important to the least important one. In the balanced realization, the fidelity of the reduced model with respect to the full model $(\mathrm{A}, \mathrm{B}, \mathrm{C})$ depends on the relation $\sigma_{\mathrm{r}} \geq \sigma_{\mathrm{r}+1}$, where $r$ is the reduced model order. Thus, the reduced state space system is given by:

$$
\begin{aligned}
& \left\{\dot{x}_{r}(t)\right\}=\left[A_{r}\right]\left\{x_{r}(t)\right\}+\left[B_{r}\right]\left\{u_{r}(t)\right\} \\
& \left\{y_{r}(t)\right\}=\left[C_{r}\right]\left\{x_{r}(t)\right\}
\end{aligned}
$$

where:

$\left\{x_{r}(t)\right\}:$ reduced state vector;

$u_{r}(t)$ : reduced input vector;

$\left[A_{r}\right]: r \times r$ reduced dynamic matrix;

$\left[B_{r}\right]: r \times m$ reduced input matrix;

$\left[C_{r}\right]: s \times r$ reduced output matrix.

For the application of balanced realization, the system represented by Eqs. 12 and 13 should be transformed into the modal domain. For this aim, the canonical state space realization was performed so that the dynamic matrix $[A]$ was transformed into modal block-diagonal matrix, whose order is $2 \times 2$ and each block corresponds to each mode of the system.

\section{CONTROL APPROACH}

Active modal control is used as control strategy for a smart composite structure as shown in Fig. 1 . In this strategy, $\delta$ is the displacement, $X$ stands the modal states, $F_{E X C}$ is the external forces and $u$ is the control effort.

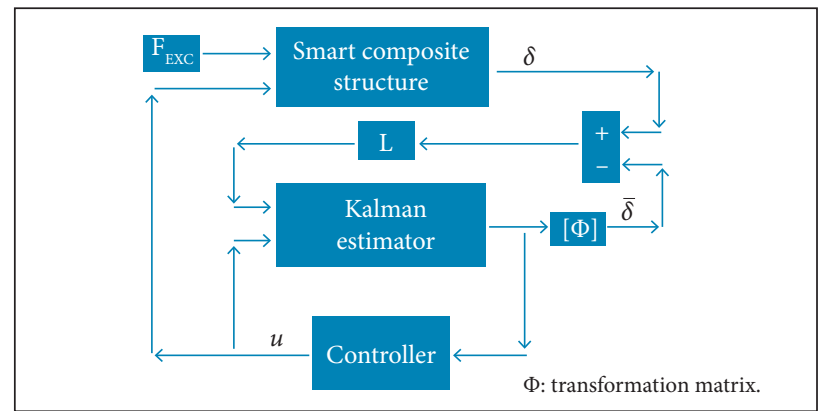

Figure 1. Active modal control based on modal state feedback control (adapted from Koroishi et al., 2014).

In the strategy shown in Fig. 1, the smart composite structure represented by Eq. 11 was written in the state space form as represented by Eqs. 12 and 13 for the simulations.

The advantage of using active modal control is that this technique is very effective for flexible structures applications, requiring a reduced number of actuators and sensors. The states are then used by the controllers to determine the control force.

The estimator is responsible for determining the modal states required by the controllers. The Kalman estimator is able to estimate the states by using noise contaminated measurement signals. More details regarding the Kalman estimator can be found in the literature (Welch and Bishop, 
1995; Anderson and Moore, 1979; Durbin and Koopman, 2002). The Kalman estimator is represented by Eq. 18:

$$
\left\{\dot{x}_{r}(t)\right\}=\left[A _ { r } \left[\left\{x_{r}(t)\right\}+\left[B_{r}\right]\{u(t)\}+[L]\{\delta(t)-\bar{\delta}(t)\}\right.\right.
$$

where:

$[L]$ : gain matrix. This matrix was determined by using the command lqe.m in the software Matlab;

$\delta(t)$ : displacement vector;

$\bar{\delta}(t)$ : estimated displacement vector.

Figure 1 shows that, in the modal state feedback control, a number of controllers are necessary. The method requires the modal displacements and modal velocities to determine the control effort of the controllers. For this purpose, the Linear Quadratic Regulator, solved by LMI, was used to determine the gains of the controllers. This method permits to take into account slight nonlinearities and uncertainties in the model.

\section{LINEAR QUADRATIC REGULATOR}

LQR has a very important role in the design of multivariable control of dynamic systems, not only as a powerful control technique, but also because it represents the source of many recently developed procedures for designing linear MIMO systems. Besides providing a methodology to control the feedback gain, the linear quadratic regulator ensures good stability margins for the closed loop system.

The optimal control, i.e. the linear quadratic regulator in the context of the present contribution, is designed, so that the minimization of the performance index leads to the optimization of pre-defined physical quantities (Ogata, 2003). Considering the feedback control given by:

$$
\{u(t)\}=-[K]\{x(t)\}
$$

the gain $[K]$ can be determined by the minimization of the performance index given by Eq. 20 .

$$
J=\int_{0}^{\infty}\{x(t)\}^{T}\left[Q_{l q r}\right]\{x(t)\}+\{u(t)\}^{T}\left[R_{l q r}\right]\{u(t)\} d t
$$

where:

$\left[Q_{l q r}\right]$ : positive defined hermitian matrix (positive definite or semi-definite) or real symmetric that weights each state;
$\left[R_{l q r}\right]$ : positive defined hermitian matrix or real symmetric that weights the energy cost of each controller (Simões, 2006).

By substituting Eq. 19 into Eq. 20, it is also possible to obtain the controller gain $[K]$.

\section{LINEAR MATRIX INEQUALITIES}

LMI is a powerful tool used in many mathematical problems. They were first presented by Aleksandr Mikhailovich Lyapunov, thus forming the well-known Lyapunov Theory (Boyd et al., 1994). He demonstrated that the differential equation:

$$
\{\dot{x}(t)\}=[A]\{x(t)\}
$$

is stable (all trajectories converge to zero), if and only if there is a positive-definite matrix $P_{l m i}$ such that:

$$
[A]^{T}\left[P_{l m i}\right]+\left[P_{l m i}\right][A]>0
$$

The inequality given by Eq. 22 is known as the Lyapunov inequality.

The advantage of using LMI for determining the controller gain is the possibility of assuming that the parameters of the model involve uncertainties.

Currently, LMI have been the object of study by many researchers around the world, aiming at different applications, such as: control of continuous and discrete systems in time domain, optimal and robust control (Van Antwerp and Braatz, 2000; Silva et al., 2004), model reductions (Assunção, 2000), control of non-linear systems, theory of robust filters (Palhares, 1998), besides detection, location and quantification of faults (Abdalla et al., 2000; Wang et al., 2007).

\section{LINEAR QUADRATIC REGULATOR USING LINEAR MATRIX INEQUALITIES}

Several authors have considered applications of LQR; however, not so many have discussed the LMI version of this controller (Johnson and Erkus, 2002). A version of LQR solved by LMI is illustrated in Erkus and Lee (2004). The authors of this contribution show that the problem LQR-LMI is described by:

$$
\min X, P_{l m i} X_{l m i} \operatorname{tr}\left(\left[Q_{l q r}\right]\left[P_{l m i}\right]\right)+\operatorname{tr}\left(\left[X_{l m i}\right]\right)+
$$

$$
\left.\operatorname{tr}\left(\left[Y_{l m i}\right] N\right)\right\}+\operatorname{tr}\left([N]^{T} Y_{l m i}{ }^{T}\right)
$$


subjected to:

$$
\begin{aligned}
& {[A][P]-[B]\left[Y_{l m i}\right]+[P][A]^{T}-\left[Y_{l m i}\right]^{T}[B]^{T}+\left[B_{w}\right]\left[B_{w}\right]^{T}<0}
\end{aligned}
$$

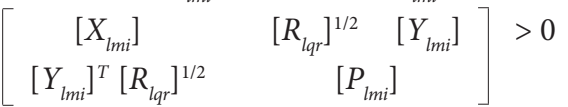

where:

$N$ : noise position vector;

$\left[X_{l m i}\right]$ and $\left[Y_{l m i}\right]$ : LMI solutions;

$\operatorname{tr}($ ): denotes the matrix trace;

$B_{w}$ : disturbance matrix.

Equation 23 was adapted from the minimization of the performance index given by Eq. 20. Then, the influence of noise was incorporated in the process of performance index minimization. By solving Eq. 23 with the constraints considered in Eq. 24, the controller gain is:

$$
[G]=\left[Y_{l m i}\right]\left[P_{l m i}\right]^{-1}
$$

\section{DESIGN OF ROBUST CONTROLLERS USING LMI}

The major advantage of LMI design is to enable specifications such as stability degree requirements, decay rate, input limitation for the actuators and output peak bounder. It is also possible to assume that the model's parameters involve uncertainties (Ogata, 2003).

The LMI is a very useful tool for problems with constraints where the parameters vary according to a range of values. The design of robust controllers used in this contribution was previously presented by Assunção and Teixeira (2001). A system with politopic uncertainties is stable if there is $[X]$ and $[G]$ such as the following LMI are feasible:

where:

$$
\begin{aligned}
{\left.\left[A_{i}\right][X] B_{i}\right][G]+[X]\left[A_{i}\right]^{T}-[G]^{T}\left[B_{i}\right]^{T}<0 } \\
{[X]>0 }
\end{aligned}
$$

$i=1,2, \ldots, m$ ( $m$ is the number of uncertainties);

$[X]$ : LMI solution.

Equation 23 was used for the robust control using LQR, and the constraints (Eq. 24) were arranged in the form given by Eq. 26.

\section{NUMERICAL SIMULATIONS}

The studied laminated composite beam, illustrated in Fig. 2, has $306 \mathrm{~mm}$ length (L), $25.5 \mathrm{~mm}$ width (b) and $1 \mathrm{~mm}$ thickness (h).

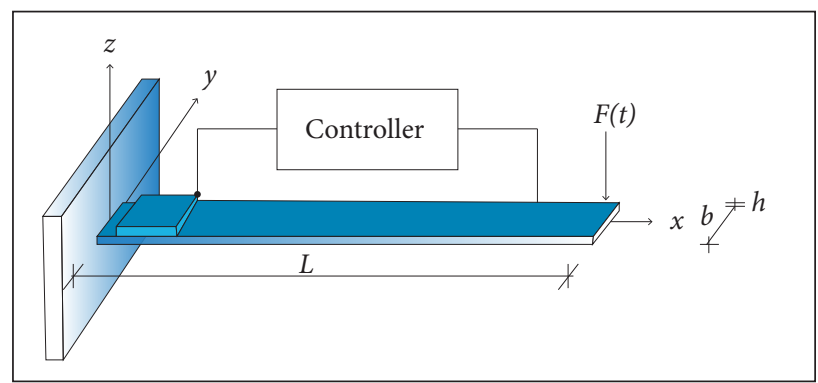

Figure 2. Composite cantilever beam with active vibration control.

A piezoelectric ceramic actuator of dimensions $45.9 \times 25.5 \mathrm{~mm}^{2}$ is bonded to the beam's top surface, $1 \mathrm{~mm}$ away from the clamp. The composite laminate has a total of 5 layers made of graphite/ epoxy and oriented as: $45^{\circ} / 0^{\circ} / 45^{\circ} / 0^{\circ} / 45^{\circ}$. The layers oriented at $0^{\circ}$ are parallel to the $x$ axis. The thicknesses of the layers are $0.2 \mathrm{~mm}$ and the thickness of the piezoceramic actuator is $1 \mathrm{~mm}$.

The constants of elastic stiffness of the beam made of AS4/3501 carbon/epoxy composite are (given in GPa): $\mathrm{C}_{11}=173.6 ; \mathrm{C}_{22}=\mathrm{C}_{33}=7.61 ; \mathrm{C}_{12}=\mathrm{C}_{13}=2.48 ; \mathrm{C}_{23}=2.31$; $\mathrm{C}_{44}=1.38 ; \mathrm{C}_{55}=\mathrm{C}_{66}=3.45$. The PZTs are: $\mathrm{C}_{11}=\mathrm{C}_{22}=\mathrm{C}_{33}=102.23$; $\mathrm{C}_{12}=\mathrm{C}_{13}=\mathrm{C}_{23}=5.035 ; \mathrm{C}_{44}=\mathrm{C}_{55}=\mathrm{C}_{66}=2.594$. The $\mathrm{PZT}$ patch piezoelectric constants are (given in $\mathrm{C} / \mathrm{m}^{2}$ ): $\mathrm{e}_{31}=-18.300$; $\mathrm{e}_{32}=\mathrm{e}_{33}=-9.013$. The electric permissivities are (given in $\mathrm{F} / \mathrm{m}$ ): $\chi_{11}=\chi_{22}=\chi_{33}=1800 \varepsilon_{0}$. The mass densities, in $\mathrm{kg} / \mathrm{m}^{3}$, are 1,578 for the composite laminated material and 7,700 for the PZT patch.

The FE model has been derived by using a $10 \mathrm{x} 1$ uniform mesh. The excitation force $(1 \mathrm{~N})$ was applied at point II and the time domain structure responses were captured at point I (see Fig. 2). The piezoelectric actuator is connected to an active control system, and the vibration amplitudes are to be minimized over time. To proceed as similar as possible to experimentation condition, band-limited white noise is superposed to the calculated displacements.

Three cases of active vibration control were analyzed:

a. Comparison between the controllers designed using Eqs. 18 and 19 and the robust controllers designed using Eqs. 22, 23 and 24. In this case, there is no uncertainty in the models, thus forming the deterministic case.

b. Robustness analyses considering variation in the model of smart composite structure so that the uncertainties were considered in the dynamical matrix $[A]+[\Delta A]$.

c. Robustness analyses considering variation in the dynamical matrix of Kalman Estimator $\left[A_{r}\right]+\left[\Delta A_{r}\right]$.

The variation applied in case (b) corresponds to possible modifications in the structure, and the variation 
considered in case (c) corresponds to uncertainties in terms of system identification. In both cases, (b) and (c), only nonparametric uncertainties were considered, and the level of these uncertainties was $10 \%$ around the nominal value. The samples of the uncertainty term $\Delta$ are determined by using Monte Carlo simulation associated with latin hypercube. The number of samples was 100 .

For the first two modes, the system was observable and controllable. For these two modes, four uncertain modes result. Table 1 presents the four uncertain model configurations (matrix $\mathrm{A}_{\mathrm{i}}$ ). These models were used to determine the robust controllers.

Table 1. Uncertain model (matrix Ai).

\begin{tabular}{|c|c|c|c|c|}
\hline \multirow{2}{*}{$A_{i}$} & \multicolumn{2}{|c|}{ Mode 1 } & \multicolumn{2}{c|}{ Mode 2 } \\
\cline { 2 - 5 } & $-10 \%$ & $+10 \%$ & $-10 \%$ & $+10 \%$ \\
\hline 1 & $X$ & -- & $X$ & -- \\
\hline 2 & $X$ & -- & -- & $X$ \\
\hline 3 & -- & $X$ & $X$ & -- \\
\hline 4 & -- & $X$ & -- & $X$ \\
\hline
\end{tabular}

\section{NUMERICAL RESULTS}

Figures 3, 4 and 5 present, respectively, the impact response, the frequency response function (FRF), and the control effort for case (a).

By analyzing these results, it is possible to observe that the system was controlled using the conventional controllers and robust controllers. The results were very similar for both controllers. The amplitude attenuation is obtained for a time smaller than $1 \mathrm{~s}$ (see Fig. 5).

The FRF is presented in Fig. 4. The first two modes were attenuated (almost $16.70 \mathrm{~dB}$ for the first frequency and 11.65 $\mathrm{dB}$ for the second frequency). Besides, no spillover effects are observed for higher modes (see Fig. 10b).

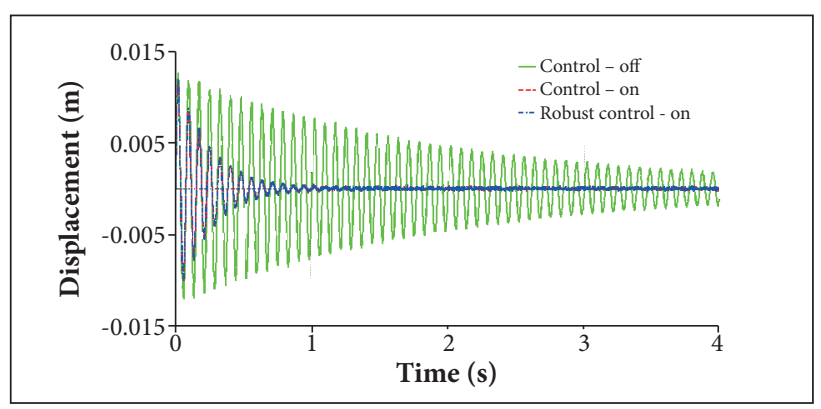

Figure 3. Impact response.
Figures 6 to 11 present results for case (b). These results are divided in two groups, namely conventional controllers (Figs. 6, 7 and 8) and robust controllers (Figs. 9, 10 and 11).

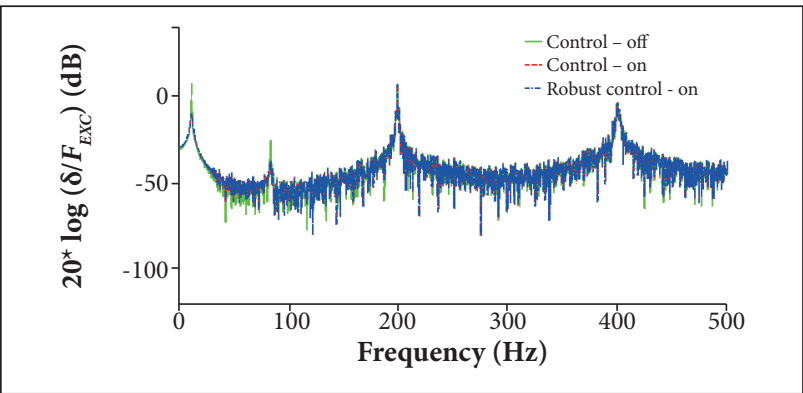

Figure 4. Frequency response function.

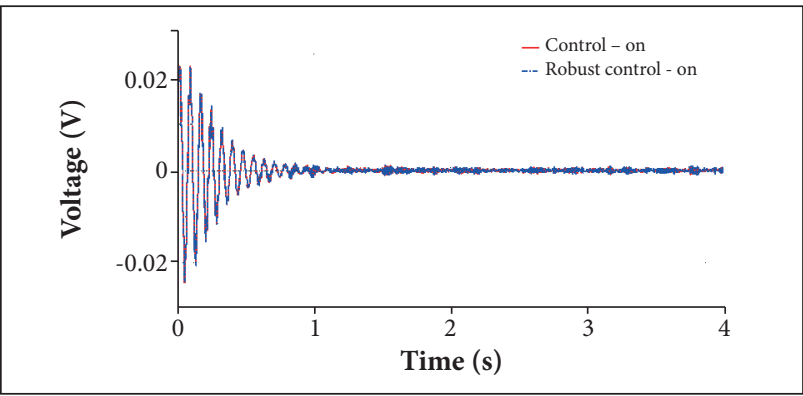

Figure 5. Control effort.

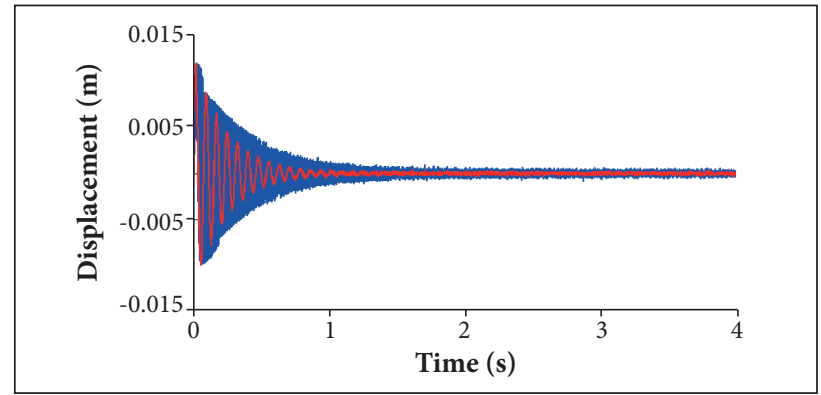

Figure 6. Impact response (case b): deterministic value (red line) and envelope (blue lines).

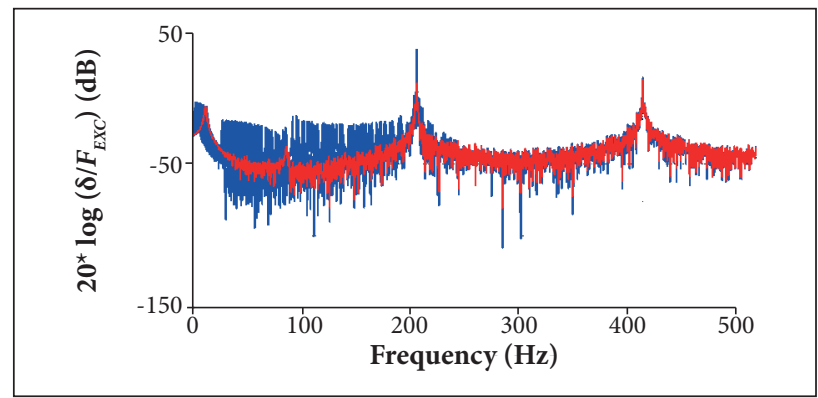

Figure 7. Frequency response function (case b): deterministic value (red line) and envelope (blue lines). 


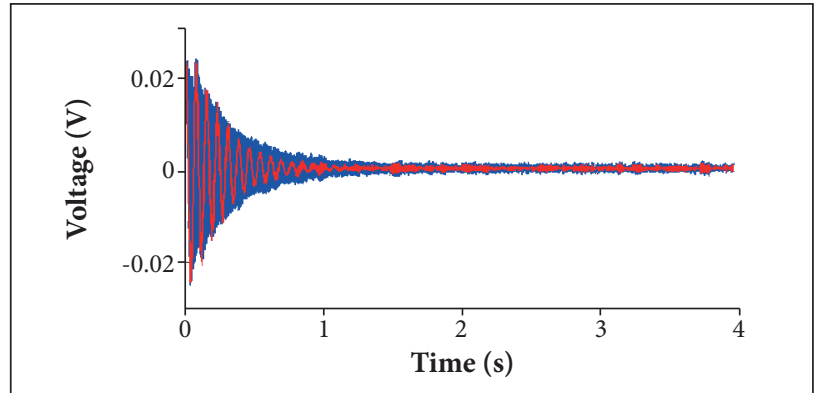

Figure 8. Control effort (case b): deterministic value (red line) and envelope (blue lines).

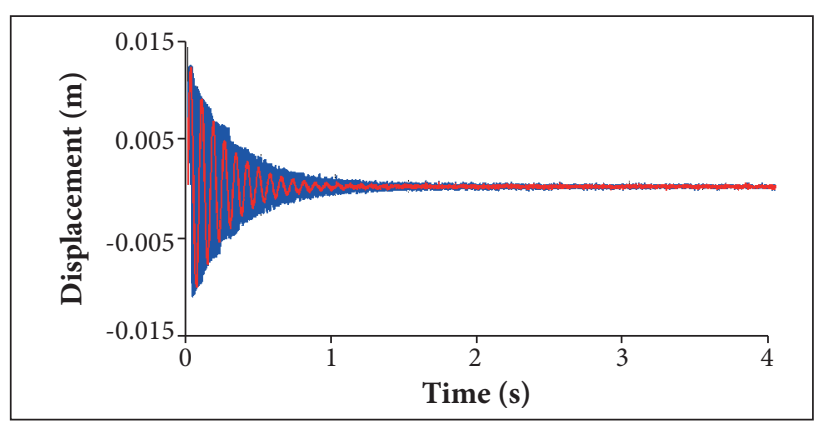

Figure 9. Impact response (case b) - robust control: deterministic value (red line) and envelope (blue lines).

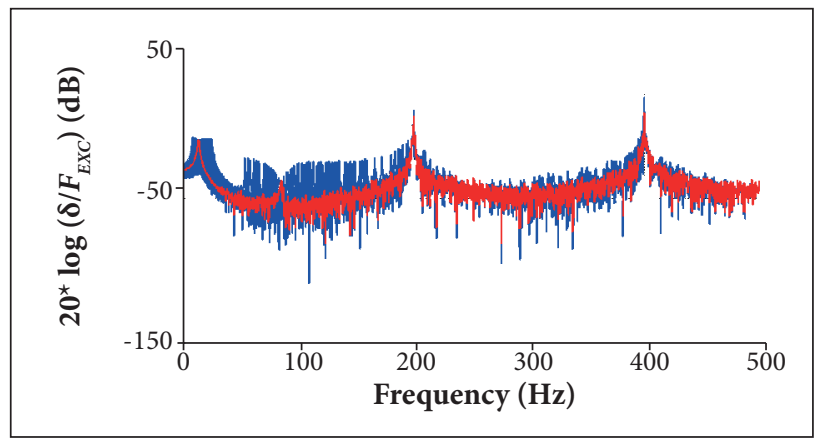

Figure 10. Frequency response function (case b) - robust control: deterministic value (red line) and envelope (blue lines).

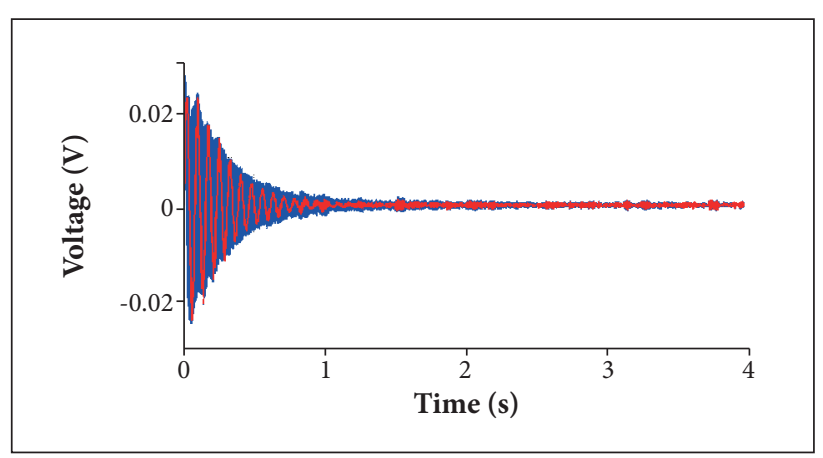

Figure 11. Control effort (case b) - robust control: deterministic value (red line) and envelope (blue lines).
In terms of impact response, Figs. 6 and 9 demonstrate that the use of robust controllers results in an envelope slightly smaller than the one obtained using conventional controllers. The same trend is observed in the control effort presented by Figs. 8 and 11. The disadvantage of using robust controllers is observed in terms of energy consumption since the control effort required when using robust controllers is bigger than the one associated with conventional controllers.

In case (b) (which considers uncertainties in the dynamical matrix of the smart composite structure), better results in terms of vibration attenuation were found (using robust controllers when the system presents variation in the model). However, these controllers lead to higher energy consumption.

Figures 12 to 17 present results for case (c), which considers uncertainty in the dynamical matrix used by the Kalman estimator. These results are divided in two groups: conventional controllers (Figs. 12, 13 and 14) and robust controllers (Figs. 15, 16 and 17).

The first aspect to point out in the results obtained for case (c) is that they present envelopes smaller than those associated with case (b) - when variation is considered in the structure's model, the natural frequency varies according to this variation, once the dynamic matrix changes with the value of $\Delta$. The same

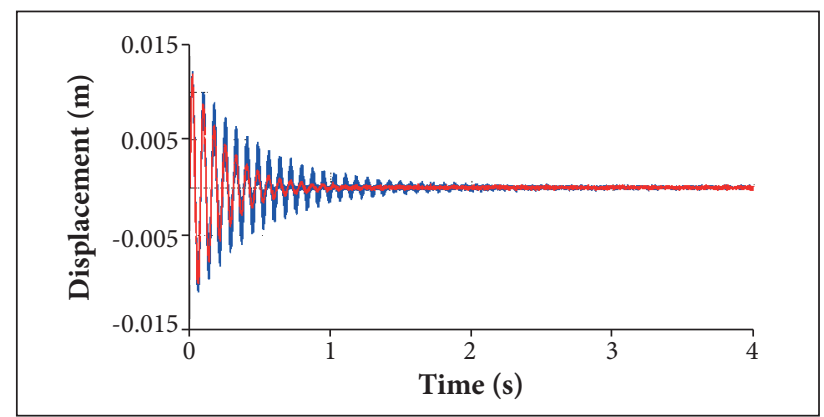

Figure 12. Impact response (case c): deterministic value (red line) and envelope (blue lines).

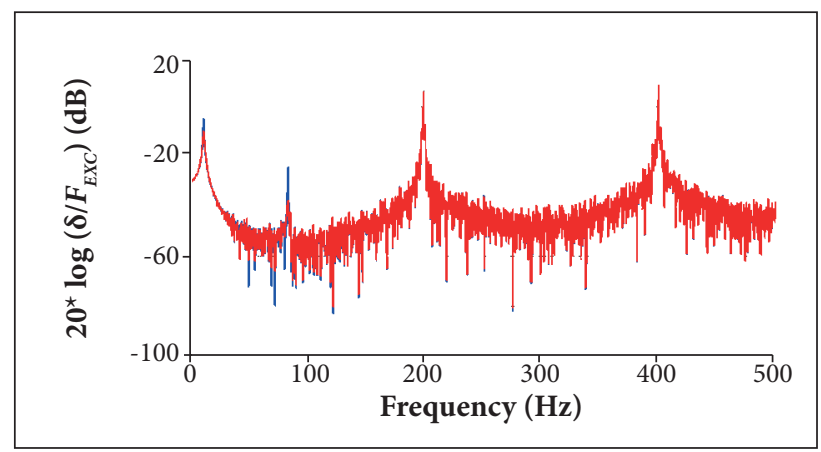

Figure 13. Frequency response function (case c): deterministic value (red line) and envelope (blue lines). 
trends are not observable in case (c), because, in this case, the variation is applied in the dynamic matrix of the estimator.

Based on the influence of the variation applied in the dynamic matrix of the estimator, it is possible to observe better results in terms of vibration attenuation when using robust controllers. Figure 15 shows that the use of robust controllers lead to a better controlled response as compared with the determinist response. However, the energy consumption increases in the first oscillations that result from the impact response (please compare Fig. 17 with Fig. 14).

A comparison between the results obtained for cases (b) and (c) was performed. This comparison was based on the

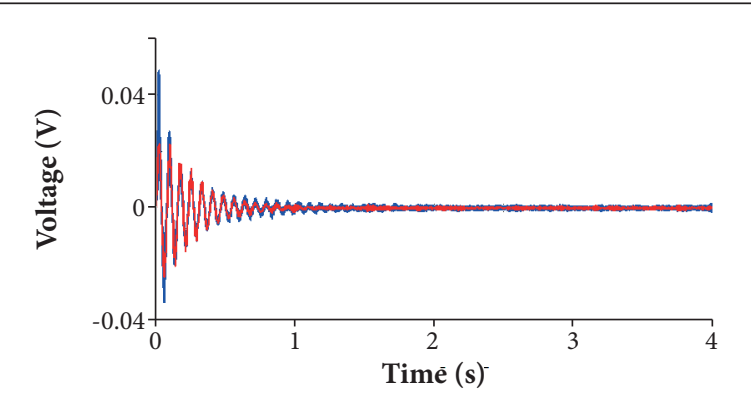

Figure 14. Control effort (case c): deterministic value (red line) and envelope (blue lines).

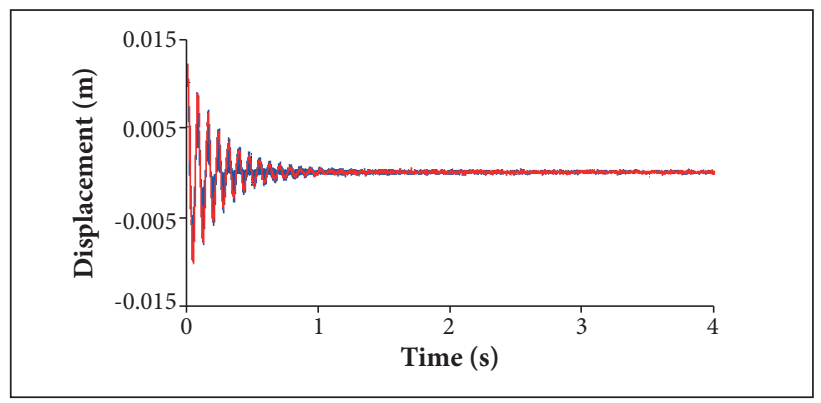

Figure 15. Impact response (case c) - robust control: deterministic value (red line) and envelope (blue lines).

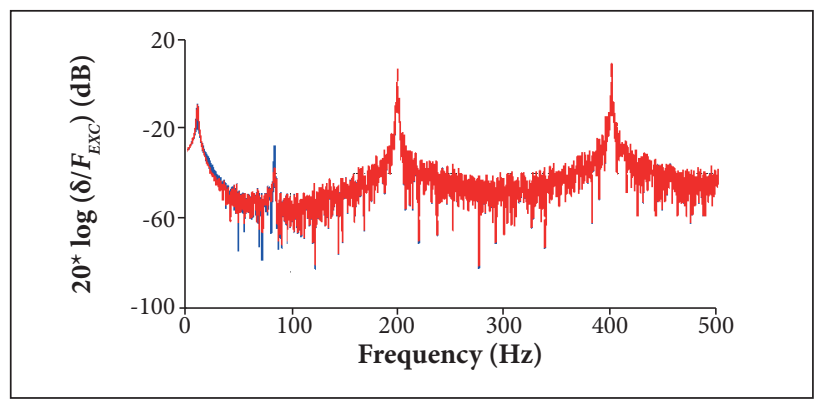

Figure 16. Frequency response function (case c) - robust control: deterministic value (red line) and envelope (blue lines). difference between the maximum and the minimum value of each envelope. Figures 18, 19 and 20 present, respectively, the interval of the controlled response, FRF, and control effort.

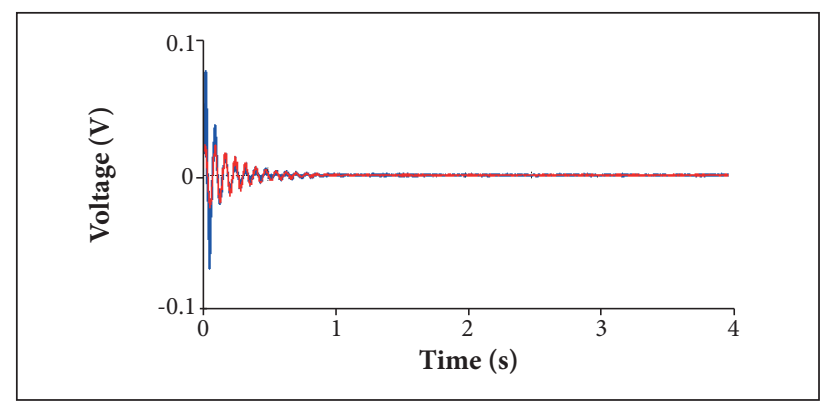

Figure 17. Control effort (case c) - robust control: deterministic value (red line) and envelope (blue lines).

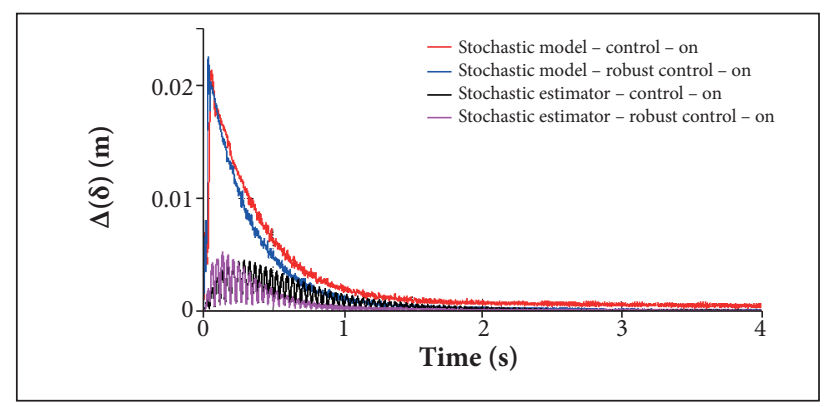

Figure 18. Difference between maximum and minimum displacement.

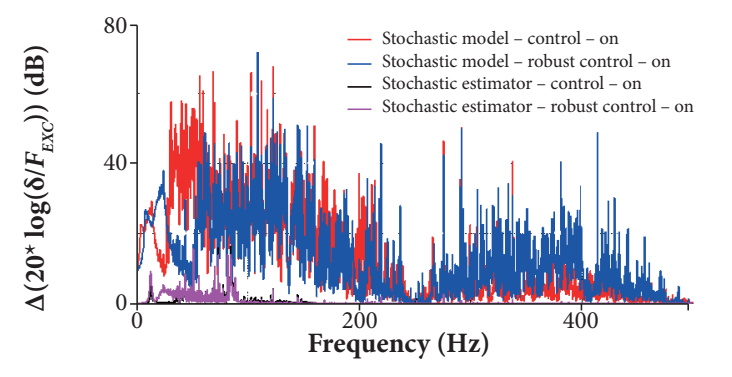

Figure 19. Difference between maximum and minimum FRF.

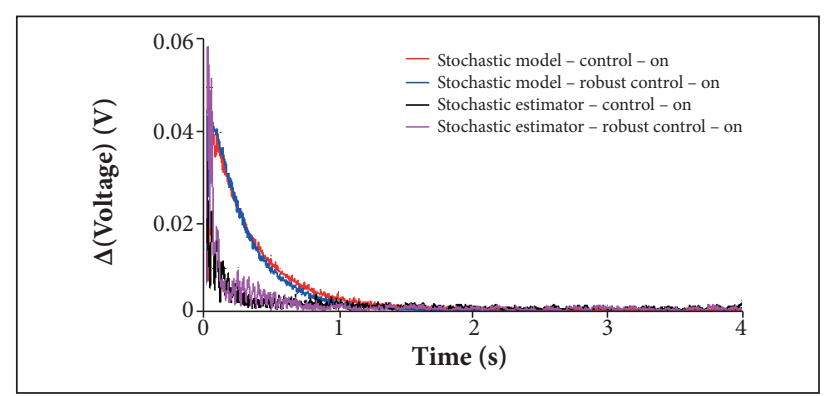

Figure 20. Difference between maximum and minimum voltage. 


\section{CONCLUSIONS}

The present contribution was dedicated to the design of robust controllers using LQR solved by LMI. The motivation for this research effort is that LMI approach represents a powerful mathematical tool that exhibits relevant characteristics which facilitate the resolution of control problems involving uncertainties. This configuration corresponds to real world applications, particularly in the case of aerospace structure design.

For illustration purposes, the present study aimed at applying the LMI technique to beams made of composite material. A model reduction method associated with the balanced realization technique was performed.

The results have revealed that the number of considered modes (two) was sufficient to achieve satisfactory control. It should be emphasized the importance of balanced realization on this stage, since this technique ranks the modes in order of relevance, regarding the dynamic behavior of the system. This means that the considered modes are the most important to the system response.

Also, with respect to the modes, the robust controller technique was effective on the first two modes and no spillover effects were observed in the higher modes. The results demonstrated the effectiveness of the methodology proposed, based on the comparison of the controlled and uncontrolled system responses. The presented results demonstrate the great potential of the proposed methodology (using LMI), since it led to very similar results as compared with those obtained via LQR solved by classical Ricatti equations. These trends were clearly observed in case (a).

Cases (b) and (c), which consider variation in the dynamic matrix of the smart composite structure and Kalman estimator, respectively, demonstrated better results in terms of vibration attenuation using robust controllers (when the system presents variation in the model). However, the robust controllers require bigger energy consumption than the conventional ones.

\section{ACKNOWLEDGEMENTS}

The authors are thankful to the Brazilian Research Agencies Fundação de Amparo à Pesquisa do Estado de Minas Gerais (FAPEMIG), Conselho Nacional de Desenvolvimento Científico e Tecnológico (CNPq) and Coordenação de Aperfeiçoamento de Pessoal de Nível Superior (CAPES) for the financial support to this work through the INCT-EIE.

\section{REFERENCES}

Abdalla, M.O., Zimmerman, D.C. and Grigoriadis, K.M., 2000, "Reduced Optimal Parameter Update in Structural Systems Using LMls", Proceedings of the 2000 American Control Conference, Chicago, USA.

Anderson, B.D.O. and Moore, J.B., 1979, "Optimal Filtering", Prentice-Hall, New Jersey, USA, 357p.

Assunção, E., 2000, "Redução H2 e Ho de Modelos através de Desigualdades Matriciais Lineares: Otimização Local e Global”, Ph.D. Thesis, Universidade Estadual de Campinas, Campinas, Brazil.

Assunção, E. and Hemerly, E.M., 1992, "Redução de Modelos de Sistemas Dinâmicos", Proceedings of the $9^{\circ}$ Congresso Brasileiro de Automática, Vol. 1, Vitória, Brasil.

Assunção, E. and Teixeira, M., 2001, "Projeto de Sistema de Controle Via LMls usando o MATLAB", Escola Brasileira de Aplicações em Dinâmica e Controle - APLICON-USP, São Carlos, Brazil.

Boyd, S., Balakrishnan, V., Feron, E. and El Ghaoui, L., 1994, "Linear Matrix Inequalities in Systems and Control Theory", Retrieved in September 06, 2015, from http://www.web.stanford.edu/ boyd/ Imibook/Imibook.pdf

Cen, S., Soh, A., Long, Y., Yao, Z., 2002, “A New 4-Node Quadrilateral FE Model with Variable Electrical Degrees of Freedom for the Analysis of Piezoelectric Laminated Composite Plates", Composite Structures, Vol. 58, No. 4, pp. 583-599. doi: 10.1016/S02638223(02)00167-8

Chee, C.Y.K., 2000, "Static Shape Control of Laminated Composite Plate Smart Structure Using Piezoelectric Actuators", Ph.D. Thesis, University of Sydney, Sydney, Australia.

Chee, C.Y.K., Tong L. and Steven, G., 2000, "A Mixed Model for Adaptive Composite Plates with Piezoelectric for Anisotropic Actuation", Computers \& Structures, Vol. 77, No. 3, pp. 253-268. doi: 10.1016/S0045-7949(99)00225-4

Conceição, S.M., Bueno, B.N., Cavalini Jr, A.A., Abreu, G.L., Melo, G.P. and Lopes Jr, V., 2009, "Model Reduction Methods for Smart Truss like Structure", Proceedings of 8th Brazilian Conference on Dynamics, Control and Applications, Bauru, Brasil.

Durbin, J. and Koopman, S.J., 2002, "Time Series Analysis by State Space Methods", Oxford University Press, Oxford, UK.

Erkus, B. and Lee, Y.J., 2004, "Linear Matrix Inequalities and Matlab LMI Toolbox", University of Southern California Group Meeting Report, Los Angeles, USA.

Johnson, E.A. and Erkus, B., 2002, "Structural Control with Dissipative Camping Devices", Proceedings of the American Control Conference, Vol. 3, Anchorage, USA. 
Koroishi, E.H., Borges, A.S., Cavalini Jr, A.A. and Steffen Jr, V., 2014 "Numerical and Experimental Modal Control of Flexible Rotor Using Electromagnetic Actuator, Mathematical Problems in Engineering, Vol. 2014, No. 2014, pp. 1-14. doi: 10.1155/2014/361418

Laub, A.J., Heath, M.T., Paige, C.C. and Ward, R.C., 1987, "Computation of Systems Balancing Transformation and Other Applications of Simultaneous Diagonalization Algorithms", IEEE Transactions on Automatic Control, Vol. 32, No. 2, pp. 115-122. doi: 10.1109/TAC.1987.1104549

Lima, A.M.G., Faria, A.W. and Rade, D.A., 2010, "Sensitivity Analysis of Frequency Response Functions of Composite Sandwich Plates Containing Viscoelastic Layers", Composites Structures, Vol. 92, No. 2, pp. 364-376. doi: 10.1016/j.compstruct.2009.08.017

Ogata, K., 2003, "Engenharia de Controle Moderno", Prentice-Hall do Brasil, São Paulo, Brazil, 788p.

Palhares, R.M., 1998, "Filtragem Robusta: uma Abordagem por Desigualdades Matriciais Lineares", Ph.D. Thesis, Universidade Estadual de Campinas, Campinas, Brazil.

Reddy, J.N., 1997, "Mechanics of Laminated Composite Plates: Theory and Analysis", Second Edition, CRC Press, London, UK.

Saravanos, D.A., Heyliger P.R. and Hopkins D.A., 1997, "Layerwise Mechanics and Finite Element for the Dynamic Analysis of Piezoelectric Composite Plates", International Journal of Solids and
Structures, Vol. 34, No. 3, pp. 359-378. doi: 10.1016/S00207683(96)00012-1

Silva, S., Lopes Jr, V. and Assunção, E., 2004, "Robust Control to Parametric Uncertainties in Smart Structures Using Linear Matrix Inequalities", Journal of the Brazilian Society of Mechanical Sciences and Engineering, Vol. 26, No. 4, pp. 430-437. doi: 10.1590/S167858782004000400008

Simões, R.C., 2006, "Controle Modal Ótimo de um Rotor Flexível Utilizando Atuadores Piezelétricos do Tipo Pilha", Ph.D., Universidade Federal de Uberlândia, Uberlândia, Brazil, 133p.

Thinh, T.I. and Ngoc, L.K., 2010, "Static Behavior and Vibration Control of Piezoelectric Cantilever Composite Plates and Comparison with Experiments", Computational Materials Science, Vol. 49, No. 4, S276-S280. doi: 10.1016/j.commatsci.2010.03.016

Van Antwerp, J.G. and Braatz, R.D., 2000, "A Tutorial on Linear and Bilinear Matrix Inequalities", Journal of Process Control, Vol. 10, No. 4, pp. 363-385. doi: 10.1016/S0959-1524(99)00056-6

Wang., H.B., Wang, J.L. and Lam, J., 2007, "Robust Fault Detection Observer Design: Iterative LMI Approaches", Journal of Dynamic Systems, Measurement, and Control, Vol. 129, No. 1, pp. 77-82. doi: 10.1115/1.2397155

Welch, G. and Bishop, G., 1995, "An Introduction to Kalman Filter, Technical Report", University of North Carolina, Chapel Hill, USA. 